\title{
Quaternionic Spatiotemporal Filtering for Dense Motion Field Estimation in Ultrasound Imaging
}

\author{
Adrien Marion (EURASIP Member), Patrick Girard, and Didier Vray (EURASIP Member)
}

Université de Lyon; CREATIS, CNRS, UMR5220; INSERM, U630; Université Lyon 1, INSA-LYON, 69621 Villeurbanne, France

Correspondence should be addressed to Adrien Marion, adrien.marion@creatis.insa-lyon.fr

Received 4 December 2009; Accepted 21 April 2010

Academic Editor: William Sandham

Copyright ( $(2010$ Adrien Marion et al. This is an open access article distributed under the Creative Commons Attribution License, which permits unrestricted use, distribution, and reproduction in any medium, provided the original work is properly cited.

Blood motion estimation provides fundamental clinical information to prevent and detect pathologies such as cancer. Ultrasound imaging associated with Doppler methods is often used for blood flow evaluation. However, Doppler methods suffer from shortcomings such as limited spatial resolution and the inability to estimate lateral motion. Numerous methods such as block matching and decorrelation-based techniques have been proposed to overcome these limitations. In this paper, we propose an original method to estimate dense fields of vector velocity from ultrasound image sequences. Our proposal is based on a spatiotemporal approach and considers $2 \mathrm{D}+\mathrm{t}$ data as a $3 \mathrm{D}$ volume. Orientation of the texture within this volume is related to velocity. Thus, we designed a bank of $3 \mathrm{D}$ quaternionic filters to estimate local orientation and then calculate local velocities. The method was applied to a large set of experimental and simulated flow sequences with low motion $(\approx 1 \mathrm{~mm} / \mathrm{s})$ within small vessels $(\approx 1 \mathrm{~mm})$. Evaluation was conducted with several quantitative criteria such as the normalized mean error or the estimated mean velocity. The results obtained show the good behaviour of our method, characterizing the flows studied.

\section{Introduction}

Blood motion estimation provides fundamental clinical information for the diagnosis of numerous pathologies such as cancer [1]. Ultrasound imaging is often used for blood flow evaluation in clinical routine. Indeed, a set of methods based on the Doppler effect are widely used to estimate blood flow velocity from ultrasound data. There are two kinds of ultrasound images, radiofrequency and B-mode. A radiofrequency (RF) ultrasound image stems from the backscattering of ultrasonic waves by a set of scatterers distributed in the tissues or the blood. B-mode images result from the demodulation of RF images, which preserves only the amplitude information. Moreover, ultrasound images are characterized by a texture, called speckle, which is related to the system's Point Spread Function (PSF). Doppler techniques are thus able to display color flow maps at a high frame rate, close to the image acquisition rate. Most notably, Kasai et al. [2] developed a real-time autocorrelation estimator that estimates the average phase shift related to the axial velocity. In addition, other methods [3-5] estimate the temporal shift instead of the phase shift. However, Doppler methods have a number of limitations. Firstly, the spatial resolution of the estimates is limited due to the use of narrowband signals. Secondly, the motion can only be estimated along the ultrasound beam axis. Therefore, it is necessary to know the angle between the velocity vector and the beam axis to estimate the velocity modulus. As a consequence, Doppler techniques are unable to estimate purely lateral motion since the axial component is null in this case.

Numerous methods have been proposed to overcome these limitations. Ferrara and Algazi [6] proposed a maximum likelihood estimator for velocity from a stochastic model of the signal from a point scatterer. It uses the effect of the scatterer velocity on both the time delay and the shift in frequency. In addition, Jensen and Munk [7] explored the use of lateral oscillations (transverse spatial modulation) to study and quantify the influence of transverse motion on the signals received. More recently, several methods proposed to make use of the statistics of the ultrasonic image by associating velocity and decorrelation $[8,9]$. Derived from 
standard Doppler approaches, Newhouse et al. [10] and then Tortoli et al. [11] suggested using a second beam to remove the angle ambiguity of conventional Doppler. In a different way, block matching techniques, called speckle tracking in ultrasonic imaging [12], have been widely studied. The principle underlying block matching techniques is based on the maximization of a similarity measure between two blocks from two successive images. To avoid decorrelation during motion, a deformation model has also been proposed [13]. Other types of methods were originally proposed by Wilson and Gill [14] to use the 3D Fourier space of an image sequence with moving objects. Recently, new estimators using this Fourier approach were proposed by Oddershede et al. [15].

Our proposal is close to the spatiofrequential approaches mentioned above. However, this paper provides a spatiotemporal development. The goal of this paper is to present an original approach based on a bank of spatiotemporal quaternionic filters to estimate apparent velocity vectors from Bmode image sequences. $2 \mathrm{D}+\mathrm{t}$ sequences can be described as $3 \mathrm{D}$ volumes. Within these volumes, local orientations of the textures due to speckle in the data volume are related to velocity. The spatiotemporal data volume $(2 \mathrm{D}+\mathrm{t})$ is filtered to estimate the local orientations of the textures. The local velocity is then deduced from this orientation. Section 2 presents the proposed methodology, and Section 3 details the data used for validation. The results obtained with various experimental and simulated ultrasound imaging flow sequences will be presented and discussed in Section 4.

\section{Quaternionic 3D Filter Banks for Motion Estimation}

Figure 1 presents the frame of reference that will be used in this paper. The $x$-axis is the lateral direction within the imaging plane, the $y$-axis is the axial direction, or propagation direction, and the $t$-axis is the temporal direction. The $z$-axis, not represented here, is the azimuthal direction.

2.1. Velocity and Orientation in Space-Time Data. An image sequence $(2 \mathrm{D}+\mathrm{t})$ can be seen as a $3 \mathrm{D}$ spatiotemporal volume. Within this volume, a pattern depends on the spatial information of the images but also on its temporal evolution. Therefore, the problem of velocity estimation can be formulated as a problem of pattern orientation.

Figure 2 illustrates an image sequence with a moving target. In this figure we introduce two angles, $\varphi$ and $\theta$, respectively associated with the velocity vector orientation within the imaging plane and the velocity modulus. The velocity vector is composed of two components $\vec{v}_{x}$ and $\vec{v}_{y}$, as illustrated in Figure 2(a). An oriented pattern, related to motion, is then presented within the plane oriented along $\vec{v}=\vec{v}_{x}+\vec{v}_{y}$ and $t$, shown in Figure 2(b).

Now, let us examine ultrasound data with motion. Figure 3 represents an ultrasound image sequence as a stack in a spatiotemporal frame of reference. This image sequence corresponds to a parabolic flow inside a cylindrical vessel.

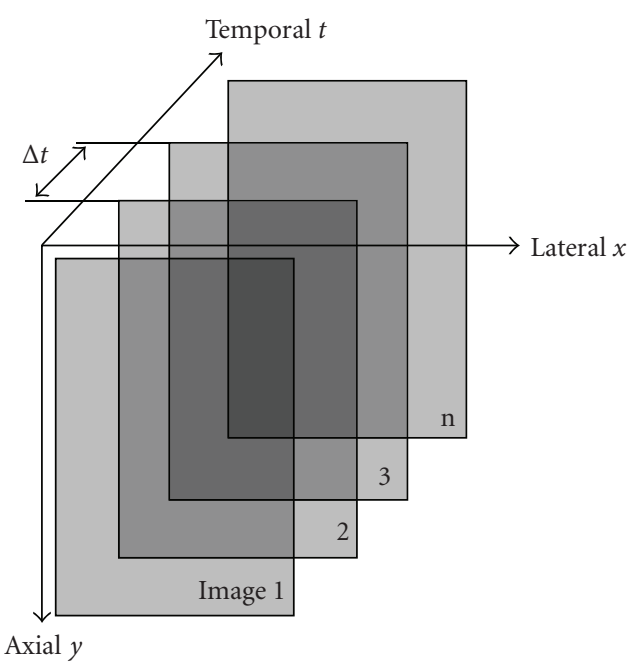

FIgURE 1: Schematic representation of an image sequence. $\Delta_{t}$ is the time interval between two successive frames.
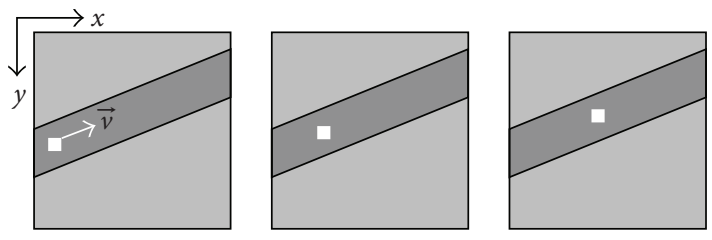

(a)

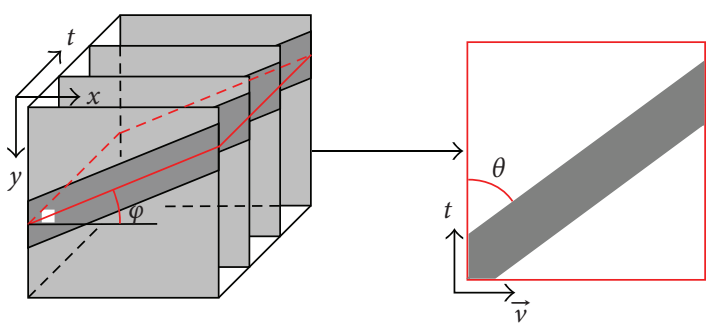

(b)

FIGURE 2: (a) Synthetic image sequence containing a moving object (white square) within the imaging plane $(x, y)$. (b) Representation of the sequence in the spatiotemporal frame of reference and a plane extracted along the velocity vector $\vec{v}$ and time $t$.

Figure 4 shows three $2 \mathrm{D}+\mathrm{t}$ representations constructed from the sequence presented in Figure 3. For different depths, the changes in the orientation of the patterns contained in the tilted spatiotemporal planes can be seen clearly. The first plane (Figure 4(a)) is located at the center of the vessel where velocity is maximum, according to the parabolic law. The second plane is near the border, where velocity is minimum, and the third plane is outside the vessel, where velocity is null.

Now, let us define the relation between orientation and velocity. According to Figure 2, the velocity modulus $|\vec{v}|=$ $\sqrt{v_{x}^{2}+v_{y}^{2}}$ is related to the angle $\theta$ following

$$
|\vec{v}|=\frac{f_{t}}{f_{s}} \tan \theta,
$$




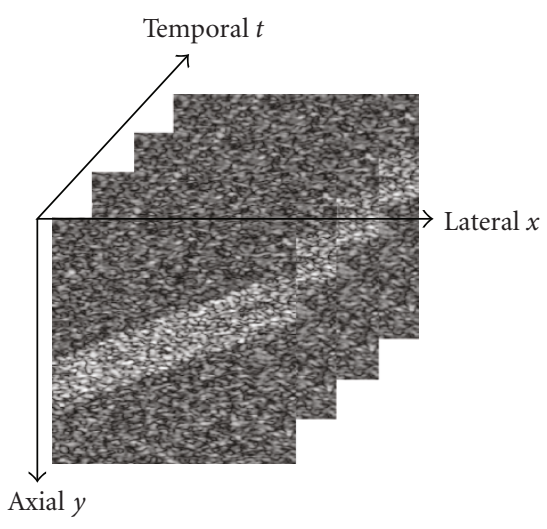

FIGURE 3: Stack of ultrasound images.

where $f_{t}$ is the temporal sampling frequency (frame rate) and $f_{s}$ the spatial sampling frequency. Then we project these results over the $x$-and $y$-axis to estimate the velocity components $v_{x}$ and $v_{y}$, as in

$$
\begin{aligned}
& v_{x}=\frac{f_{t}}{f_{x}} \tan \theta \cdot \cos \varphi, \\
& v_{y}=\frac{f_{t}}{f_{y}} \tan \theta \cdot \sin \varphi,
\end{aligned}
$$

where $f_{t}$ is the frame rate, $f_{x}$ the spatial sampling frequency along the lateral direction, and $f_{y}$ the spatial sampling frequency along the axial direction. If necessary, further information is given in [16] on the relation between the orientation in $2 \mathrm{D}+\mathrm{t}$ volume and velocity.

\subsection{Spatiotemporal-Oriented Filters for Motion}

Estimation. Equations (1), (2), and (3) show that vector velocity can be estimated from the texture angles $\theta$ and $\varphi$. We know from [16] that the use of a 3D anisotropic Gaussian filter is suitable to estimate orientations from $2 \mathrm{D}+\mathrm{t}$ ultrasound moving data. In this paper, we propose to develop a bank of 3D quaternionic filters to estimate local orientations and then provide estimated dense motion fields. First, we defined a bank of oriented filters and a region of interest (ROI). Next, each of the filters was convolved with the ROI and then the energy criteria were calculated from each filtered data to estimate the main local orientations. In the following subsections, we will detail the design of $3 \mathrm{D}$ filters then define the energy criterion.

2.2.1. Construction of $3 D$-Oriented Filters. Firstly, we defined a 3D-Gaussian filter initially aligned along the $x$-axis. Secondly, the bank of filters was constructed by successive rotations of the original filter in the three-dimensional space. According to Figure 2, the oriented filter is subjected to a rotation $R_{\varphi, t}$ with an angle $\varphi$ around the $t$-axis then to a rotation $R_{\theta, y^{\prime}}$ with an angle $\theta$ around the $y^{\prime}=R_{\varphi, t}(y)$-axis. It is similar to estimating the velocity vector orientation $\varphi$ within the plane $(x, y)$, and then the $2 \mathrm{D}$ velocity modulus, related to the orientation $\theta$. This methodology implies the use of moving axes because the second rotation is performed around the $y^{\prime}$-axis, which is the $y$-axis rotated at an angle $\varphi$ around the $t$-axis. Consequently, we propose to use quaternion algebra to develop our bank of oriented filters. Quaternion modelling provides precise and fast framework for modelling rotations; it outperforms approaches based on Euler angles, and also does not suffer from ambiguities such as Gimbal lock.

Let us recall the definitions and properties of quaternions. A quaternion is a linear combination of unitary elements and can be written as in

$$
Q=a+b \cdot i+c \cdot j+d \cdot k,
$$

where $a, b, c, d \in \mathbb{R}^{4}$ and $i, j, k \in \mathbb{C}^{3}$.

Another simpler formulation is given in

$$
Q=(a, \vec{V}),
$$

where $a \in \mathbb{R}$ and $\vec{V}=(b, c, d) \in \mathbb{R}^{3}$. $a$ is called the scalar component and $\vec{V}$ the vectorial component.

Based on these definitions, we define a rotation $R_{2 \psi, \vec{V}_{n}}$ with an angle $2 \psi$ around the $\vec{V}_{n}$-axis. The coordinates of $\vec{U}^{\prime}$, the image of vector $\vec{U}$ after the rotation $R_{2 \psi, \vec{V}_{n}}$, are given in

$$
\begin{aligned}
\left(0, \vec{U}^{\prime}\right)= & \left(0, R_{2 \psi, \vec{V}_{n}}(\vec{U})\right) \\
= & \left(\cos (\psi), \sin (\psi) \vec{V}_{n}\right) \cdot(0, \vec{U}) \\
& \cdot\left(\cos (\psi),-\sin (\psi) \vec{V}_{n}\right),
\end{aligned}
$$

where $\left(\cos (\psi), \sin (\psi) \vec{V}_{n}\right)$ and $\left(\cos (\psi),-\sin (\psi) \vec{V}_{n}\right)$ are two unitary conjugate quaternions, while $\left(0, \vec{U}^{\prime}\right)$ and $(0, \vec{U})$ are two quaternions whose scalar component is null.

Based on the formula expressed in (6), we constructed a bank of spatiotemporal filters by defining the range for $\theta$ and $\varphi$ and the angular resolution. Each 3D-oriented Gaussian filter is defined in (7):

$$
g_{\varphi, \theta}(x, y, t)=\frac{1}{(2 \pi)^{3 / 2} \sigma_{x} \sigma_{y} \sigma_{t}} \exp \left(-\frac{x^{\prime \prime 2}}{2 \sigma_{x}^{2}}-\frac{y^{\prime \prime 2}}{2 \sigma_{y}^{2}}-\frac{t^{\prime \prime 2}}{2 \sigma_{t}^{2}}\right),
$$

where $\left(x^{\prime \prime}, y^{\prime \prime}, t^{\prime \prime}\right)$ are the coordinates after rotations of $\theta$ and $\varphi$. When $\theta$ and $\varphi$ are null, the filter is aligned along the $x$-axis.

Thus, it was necessary to bring the new frame of reference $\left(x^{\prime \prime}, y^{\prime \prime}, t^{\prime \prime}\right)$ toward the initial one $(x, y, t)$. To achieve this, we performed inverse rotations, that is, a rotation $R_{-\theta, y}$ with an angle $-\theta$ around the $y$-axis then a rotation $R_{-\varphi, t^{\prime}}$ with an angle $-\varphi$ around the $t^{\prime}=R_{-\theta, y}(t)$-axis. This was done following three steps.

First, we defined the first quaternion $Q_{1}$ corresponding to the rotation $R_{-\theta, y}$ in

$$
Q_{1}=\cos \left(\frac{-\theta}{2}\right)+\sin \left(\frac{-\theta}{2}\right) j
$$

because $\vec{V}_{n}=(0,1,0)$. 


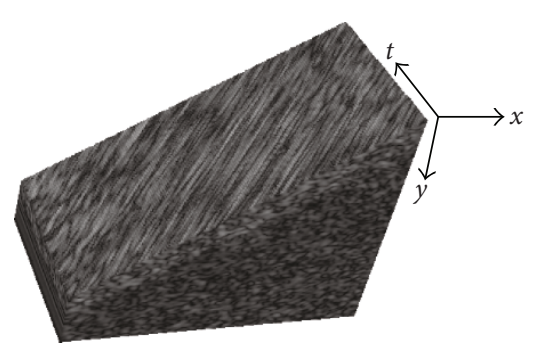

(a)

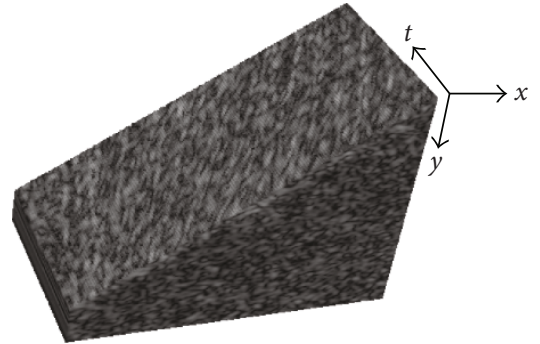

(b)

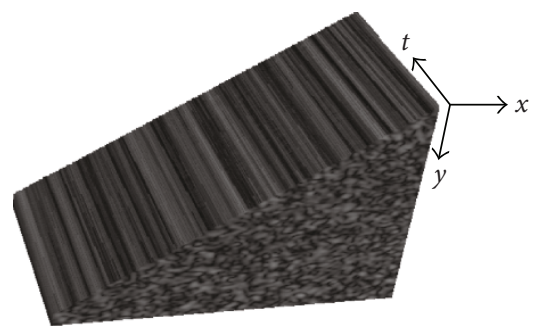

(c)

FIGURE 4: Tilted spatiotemporal planes extracted from the $2 \mathrm{D}+\mathrm{t}$ data volume at several depths: (a) at the center of the vessel (maximum velocity), (b) close to the border of the vessel (minimum velocity), (c) outside the vessel (no velocity).

From $Q_{1}$, we calculated in (9) the intermediate coordinates $\vec{U}^{\prime}=\left(x^{\prime}, y^{\prime}, t^{\prime}\right)$ using the definition given in (6):

$$
\begin{aligned}
\left(0, \vec{U}^{\prime}\right)= & \left(\cos \left(\frac{-\theta}{2}\right)+\sin \left(\frac{-\theta}{2}\right) j\right) \cdot(0, \vec{U}) \\
& \cdot\left(\cos \left(\frac{-\theta}{2}\right)-\sin \left(\frac{-\theta}{2}\right) j\right) .
\end{aligned}
$$

Second, we calculated in (10) the coordinates of vector $\vec{t}^{\prime}$, the modified vector of $\vec{t}$ with $R_{-\theta, y}$ :

$$
\begin{aligned}
\left(0, \vec{t}^{\prime}\right)= & \left(\cos \left(\frac{-\theta}{2}\right)+\sin \left(\frac{-\theta}{2}\right) j\right) \cdot(0, \vec{t}) \\
& \cdot\left(\cos \left(\frac{-\theta}{2}\right)-\sin \left(\frac{-\theta}{2}\right) j\right),
\end{aligned}
$$

where $\vec{t}=(0,0,1)$.

Next, we used vector $\overrightarrow{t^{\prime}}$ to define the second quaternion $Q_{2}$ in

$$
\begin{aligned}
Q_{2}= & \cos \left(\frac{-\varphi}{2}\right)+\sin \left(\frac{-\varphi}{2}\right) \vec{t}^{\prime}(1) \\
& +\sin \left(\frac{-\varphi}{2}\right) \vec{t}^{\prime}(2)+\sin \left(\frac{-\varphi}{2}\right) \vec{t}^{\prime}(3) .
\end{aligned}
$$

Third, the coordinates of $\vec{U}^{\prime \prime}=\left(x^{\prime \prime}, y^{\prime \prime}, t^{\prime \prime}\right)$ were obtained from the formula in (12):

$$
\begin{aligned}
\left(0, \vec{U}^{\prime \prime}\right)= & \left(\cos \left(\frac{-\varphi}{2}\right), \sin \left(\frac{-\varphi}{2}\right) \vec{t}^{\prime}\right) \cdot\left(0, \vec{U}^{\prime}\right) \\
& \cdot\left(\cos \left(\frac{-\varphi}{2}\right),-\sin \left(\frac{-\varphi}{2}\right) \vec{t}^{\prime}\right) .
\end{aligned}
$$

Finally, banks of 3D spatiotemporal oriented filters were constructed using this methodology. Figure 5 illustrates a set of four examples of 3D filters with the following characteristics:

$$
\begin{gathered}
(\varphi, \theta)=\left(0^{\circ}, 0^{\circ}\right),\left(30^{\circ}, 0^{\circ}\right),\left(30^{\circ}, 50^{\circ}\right),\left(30^{\circ}, 90^{\circ}\right), \\
\sigma_{x}=10, \\
\sigma_{y}=1, \\
\sigma_{t}=1 .
\end{gathered}
$$

From a practical point of view, if a good accuracy is required, one must use more than four filters. We can choose, for example, $\theta \in[0, \pi / 2]$ with a step equal to $2^{\circ}$, and $\varphi \in$ $[0, \pi / 2]$ with a step equal to $5^{\circ}$.

2.2.2. Energy Criterion. After having convolved the spatiotemporal volume with each of the 3D filters, we had to choose which filter brought the maximum response in order to determine the best orientation. We defined an energy criterion to determine the best candidate filter. Let us define $I_{\varphi, \theta}$ as the values of the volume filtered by the kernel whose orientations are $(\varphi, \theta)$. The criterion is then calculated for each pixel of the $2 \mathrm{D}+\mathrm{t}$ volume using a neighbourhood composed of $C$ columns, $R$ rows, and $F$ frames. For each voxel of a filtered volume and for each orientation $(\varphi, \theta)$, we calculated the criterion defined in (14):

$$
\begin{aligned}
& E(x, y, t, \varphi, \theta) \\
& =\sum_{c=-C / 2}^{C / 2} \sum_{r=-R / 2}^{R / 2} \sum_{f=-F / 2}^{F / 2}\left(I_{\varphi, \theta}(x+c, y+r, t+f)-\overline{I_{\varphi, \theta}}(x, y, t)\right)^{2},
\end{aligned}
$$




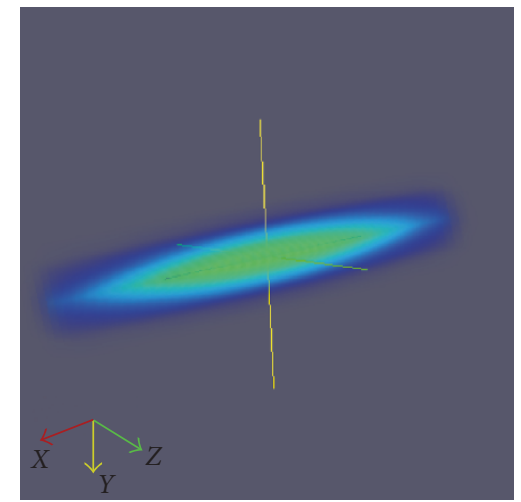

(a) $\left(\varphi=0^{\circ} \theta=0^{\circ}\right)$

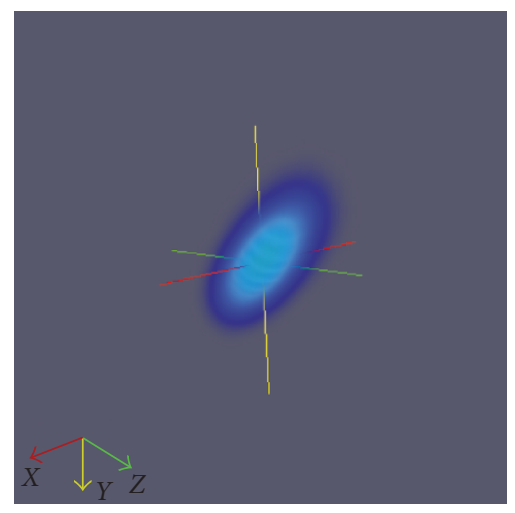

(c) $\left(\varphi=30^{\circ} \theta=50^{\circ}\right)$

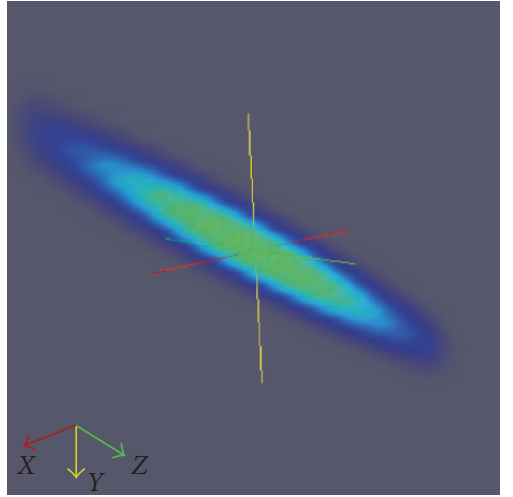

(b) $\left(\varphi=30^{\circ} \theta=0^{\circ}\right)$

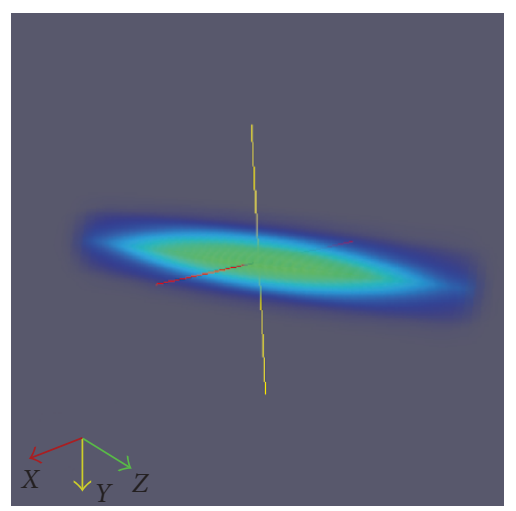

(d) $\left(\varphi=30^{\circ} \theta=90^{\circ}\right)$

Figure 5: Representation of 3D filters for different pairs $(\varphi, \theta)$

where $\overline{I_{\varphi, \theta}}(x, y, t)$ is the mean of the values $I_{\varphi, \theta}$ over the neighbourhood of size $C * R * F$ voxels centered in $(x, y, t)$.

The principal orientation was then determined by maximizing, according to $\varphi$ and $\theta$, the quantity $E(x, y, t, \varphi, \theta)$, as expressed in

$$
\widehat{(\varphi, \theta)}=\arg \max _{\varphi, \theta}(E(x, y, t, \varphi, \theta)) .
$$

The shape of this criterion, as a function of $\varphi$ and $\theta$, is represented in Figure 6.

From the estimated orientations $(\varphi, \theta)$, we calculated a local velocity measure from (2)-(3). Finally, the motion estimation algorithm can be summarized in five steps:

(1) definition of the 3D ROI whose velocity is sought,

(2) definition of the range values for $\varphi$ and $\theta\left(N_{\varphi}\right.$ and $N_{\theta}$ are, respectively, the number of $\varphi$ angles and $\theta$ angles),

(3) computation of the $\left(N_{\theta} * N_{\varphi}\right) 3 \mathrm{D}$ oriented filters $g_{\varphi, \theta}(x, y, t)$

(4) filtering of the 3D ROI with each of the filters to obtain $\left(N_{\theta} * N_{\varphi}\right) 3$ D filtered volumes,

(5) for each voxel $(x, y, t)$,

(a) computation of the criterion $E(x, y, t, \varphi, \theta)$ for each filtered volume, (b) selection of the best pair $(\varphi, \theta)$ by maximizing the energy criterion,

(c) computation of the local vector velocity according to (2) and (3).

\section{Data Used for the Evaluation}

This section presents the data used for the evaluation of the algorithm to estimate local velocities. This work focused on high-frequency, $40 \mathrm{MHz}$ imaging because Doppler techniques are not efficient with these data. In high-frequency, $40 \mathrm{MHz}$ imaging, arrays are not yet implemented and these systems use a single transducer continuously moving for imaging. Thus, Doppler techniques necessitate stopping the transducer to provide velocity estimates that is a strong limitation. So we decided to use high-frequency, $40 \mathrm{MHz}$ simulated and experimental data with various velocities and orientations for the evaluation of our method. Orientation is defined, within the imaging plane, as the angle between the lateral axis $(x)$ and the flow ( $\varphi$ in Figure 2(b)). The velocity is defined by the mean value inside the vessel, which is equal to $66 \%$ of the maximum value according to the theoretical parabolic profile.

3.1. Simulated Data. The simulation of blood flow sequences was based on the system approach of Meunier and Bertrand 


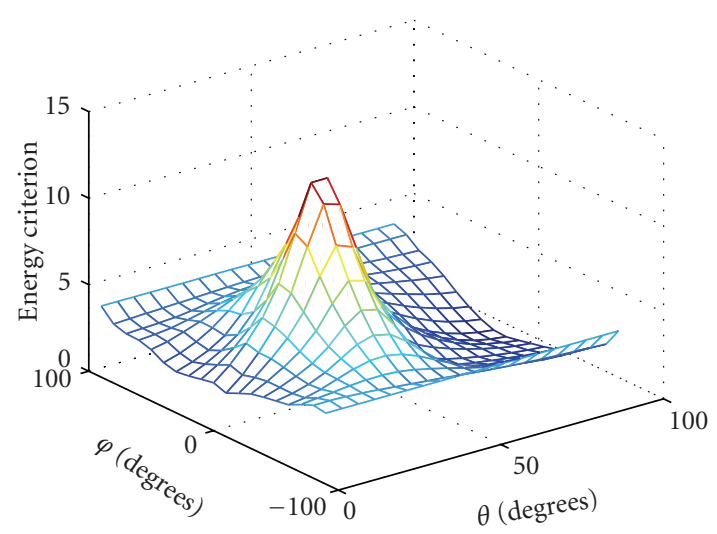

Figure 6: Example of an energy criterion curve as a function of $\varphi$ and $\theta$.

[17]. For a realistic simulation, a 3D set of scatterers was generated and then displaced following a paraboloid law. The diameter of the vessel was equal to $1 \mathrm{~mm}$. An out-ofplane angle equal to $5^{\circ}$ was used to simulate the out-of-plane motion that occurs in real situations. The convolution was performed using a numerical convolution algorithm after approximation of the scatterers to nodes of a sampled grid. Radiofrequency (RF) images were demodulated and then log-compressed to create sequences of B-mode images at 30 frames per second. Central frequency was set to $40 \mathrm{MHz}$. Axial and lateral resolutions were equal to $40 \mu \mathrm{m}$ and $80 \mu \mathrm{m}$, respectively. Further information on the simulation methodology can be found in [18].

We used 16 experimental sequences with mean velocities ranging from $0.4 \mathrm{~mm} / \mathrm{s}$ to $2 \mathrm{~mm} / \mathrm{s}$ and orientations from $0^{\circ}$ to $30^{\circ}$. We chose orientations in this range because Doppler techniques are unable to provide reliable estimates for these cases since only the axial projection of the velocity is estimated with Doppler. The prefix $S_{s}$ was added to this set of sequences.

3.2. Experimental Data. We also used experimental data to validate our method. These data were acquired from circulation of a fluid in a phantom.

3.2.1. Phantom and Blood-Mimicking Fluid. A phantom in gelatin was used to simulate the biological tissues. Silica was added to the phantom to replace scatterers. A blood vessel, $1 \mathrm{~mm}$ in diameter, was placed inside the phantom. As described in [19], blood-mimicking fluid was used as the circulation medium in the vessel. The fluid consists of $5 \mu \mathrm{m}$ diameter nylon scattering particles (Orgasol, ELF, Atochem, Paris, France) suspended in a fluid base of water, glycerol, dextran, and surfactant. The fluid was filtered and degassed before being injected into the flow circuit setup. Ramnarine et al. [19] showed that the fluid's characteristics (density, viscosity, ultrasound propagation velocity and attenuation) were close to the characteristics of human blood. The bloodmimicking fluid was injected into the vessel using a motorcontrolled pump (Pump 11, Harvard Apparatus). The mean velocity was less than $2 \mathrm{~mm} / \mathrm{s}$.
TABLE 1: List of the flow sequences used for the validation.

\begin{tabular}{|c|c|c|c|}
\hline Name & Type & Orientation $\varphi$ & $v_{\text {mean }}$ \\
\hline$S_{f b 1}$ & Experimental & $0^{\circ}$ & $0.25 \mathrm{~mm} / \mathrm{s}$ \\
\hline$S_{f b 2}$ & Experimental & $0^{\circ}$ & $0.5 \mathrm{~mm} / \mathrm{s}$ \\
\hline$S_{f b 3}$ & Experimental & $0^{\circ}$ & $1 \mathrm{~mm} / \mathrm{s}$ \\
\hline$S_{f b 4}$ & Experimental & $0^{\circ}$ & $2 \mathrm{~mm} / \mathrm{s}$ \\
\hline$S_{f s 1}$ & Experimental & $8^{\circ}$ & $0.37 \mathrm{~mm} / \mathrm{s}$ \\
\hline$S_{f s 2}$ & Experimental & $8^{\circ}$ & $0.53 \mathrm{~mm} / \mathrm{s}$ \\
\hline$S_{f s 3}$ & Experimental & $8^{\circ}$ & $0.83 \mathrm{~mm} / \mathrm{s}$ \\
\hline$S_{f_{s 4}}$ & Experimental & $8^{\circ}$ & $1.73 \mathrm{~mm} / \mathrm{s}$ \\
\hline$S_{s 1}$ & Simulated & $0^{\circ}$ & $0.4 \mathrm{~mm} / \mathrm{s}$ \\
\hline$S_{s 2}$ & Simulated & $0^{\circ}$ & $0.8 \mathrm{~mm} / \mathrm{s}$ \\
\hline$S_{s 3}$ & Simulated & $0^{\circ}$ & $1 \mathrm{~mm} / \mathrm{s}$ \\
\hline$S_{s 4}$ & Simulated & $0^{\circ}$ & $2 \mathrm{~mm} / \mathrm{s}$ \\
\hline$S_{s 5}$ & Simulated & $10^{\circ}$ & $0.4 \mathrm{~mm} / \mathrm{s}$ \\
\hline$S_{s 6}$ & Simulated & $10^{\circ}$ & $0.8 \mathrm{~mm} / \mathrm{s}$ \\
\hline$S_{s 7}$ & Simulated & $10^{\circ}$ & $1 \mathrm{~mm} / \mathrm{s}$ \\
\hline$S_{s 8}$ & Simulated & $10^{\circ}$ & $2 \mathrm{~mm} / \mathrm{s}$ \\
\hline$S_{s 9}$ & Simulated & $20^{\circ}$ & $0.4 \mathrm{~mm} / \mathrm{s}$ \\
\hline$S_{s 10}$ & Simulated & $20^{\circ}$ & $0.8 \mathrm{~mm} / \mathrm{s}$ \\
\hline$S_{s 11}$ & Simulated & $20^{\circ}$ & $1 \mathrm{~mm} / \mathrm{s}$ \\
\hline$S_{s 12}$ & Simulated & $20^{\circ}$ & $2 \mathrm{~mm} / \mathrm{s}$ \\
\hline$S_{s 13}$ & Simulated & $30^{\circ}$ & $0.4 \mathrm{~mm} / \mathrm{s}$ \\
\hline$S_{s 14}$ & Simulated & $30^{\circ}$ & $0.8 \mathrm{~mm} / \mathrm{s}$ \\
\hline$S_{s 15}$ & Simulated & $30^{\circ}$ & $1 \mathrm{~mm} / \mathrm{s}$ \\
\hline$S_{s 16}$ & Simulated & $30^{\circ}$ & $2 \mathrm{~mm} / \mathrm{s}$ \\
\hline
\end{tabular}

We used eight experimental sequences with different situations of velocity and orientation. Among them, four sequences were carried out with blood-mimicking fluid and four with true rabbit blood. The mean velocities were included in the range of $[0.25 \mathrm{~mm} / \mathrm{s}, 2 \mathrm{~mm} / \mathrm{s}]$, whereas orientation was set in the range of $\left[0^{\circ}, 8^{\circ}\right]$. The prefix $S_{f b}$ was added to the first set of sequences while the prefix $S_{f s}$ was added to the second set of sequences.

3.2.2. Ultrasound Imaging System. An ultrasonic system (Vevo 660, VisualSonics, Totonto) operating at $40 \mathrm{MHz}$ was used to acquire B-mode sequences of 300 images at 30 frames per second. Axial and lateral resolutions were equal to $40 \mu \mathrm{m}$ and $80 \mu \mathrm{m}$, respectively. Figure 7 shows a 3D view of the spatiotemporal volume with three orthogonal slices.

\subsection{Synthesis. Table 1 contains all the sequences used for our evaluation. \\ Let us note that all data are temporal image sequences that are manipulated as 3D volumes. The size of voxels depends on the imaging system; it is equal to $15.6 \mu \mathrm{m}$ along spatial dimensions ( $x$ - and $y$-axis) and $33 \mathrm{~ms}$ along temporal dimension ( $t$-axis). In the following, we will equally mention displacements in pixels per frame or blood velocity in millimeters per second.}




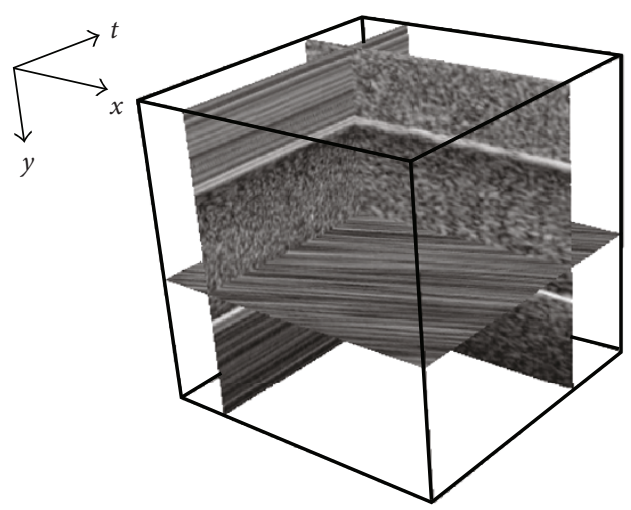

FIGURE 7: 3D view of the spatiotemporal volume from an experimental US image sequence.

\section{Evaluation of the Method}

The proposed method was applied to each of the sequences defined in Table 1. For each sequence, we computed dense velocity fields within an ROI, centered on the vessel. An ROI example is illustrated in Figure 8. Estimates were calculated for 15 successive images. From these estimates, we extracted the column located at the center of the ROI then calculated means and standard deviations along the 15 temporal columns, as schematized in Figure 8. Thus, for each sequence, we had two velocity profiles for $x$ and $y$. The mean estimates were called $\widehat{v_{x_{i}}}$ and $\widehat{v_{y_{i}}}$, whereas the mean standard deviations were called $s t d_{x_{i}}$ and $s t d_{y_{i}}$. Then we defined several performance criteria to quantify the accuracy of the estimator. Some of them have been used by researchers proposing new vector velocity estimation methods in flow imaging $[15,20]$.

\subsection{Performance Criteria}

4.1.1. Normalized Mean Errors along $x$ and $y$. These criteria evaluate, for each estimate of $v_{x}$ and $v_{y}$, the mean errors compared with the theoretical profiles. We defined them in (16):

$$
\overline{E_{x}}=\frac{1}{N} \sum_{i=1}^{N}\left|\frac{\widehat{v_{x_{i}}}-v_{x_{i}}}{v_{x_{\max }}}\right|, \quad \overline{E_{y}}=\frac{1}{N} \sum_{i=1}^{N}\left|\frac{\widehat{v_{y_{i}}}-v_{y_{i}}}{v_{y_{\max }}}\right|,
$$

where $v_{x_{i}}$ and $v_{y_{i}}$ are the theoretical velocities according to the parabolic profiles and $v_{x_{\max }}$ and $v_{y_{\max }}$ the maximum theoretical velocities along $x$ and $y$.

4.1.2. Normalized Mean Standard Deviation along $x$ and $y$. These standard deviations quantify the variability of estimates and are defined in (17):

$$
\overline{s t d_{x}}=\sqrt{\frac{1}{N} \sum_{i=1}^{N} \frac{s t d_{x_{i}}^{2}}{v_{x_{\max }}}}, \quad \overline{s t d_{y}}=\sqrt{\frac{1}{N} \sum_{i=1}^{N} \frac{s t d_{y_{i}}^{2}}{v_{y_{\max }}},}
$$

where $s t d_{x_{i}}^{2}$ and $s t d_{y_{i}}^{2}$ are the temporal variances of estimates $v_{x_{i}, y_{i}}$ at depth $i$.
4.1.3. Mean Velocity Components along $x$ and $y$. The mean velocity components within the vessel are examined in order to evaluate the global behaviour of the estimator. The mean velocity components were obtained from (18):

$$
\widehat{v_{x}}=\frac{1}{N} \sum_{i=1}^{N} \widehat{v_{x_{i}}}, \quad \widehat{v_{y}}=\frac{1}{N} \sum_{i=1}^{N} \widehat{v_{y_{i}}} .
$$

4.1.4. Mean Velocity Modulus. From components $\widehat{v_{x_{i}}}$ and $\widehat{v_{y_{i}}}$, we calculated the mean velocity modulus in (19):

$$
\widehat{|v|}=\frac{1}{N} \sum_{i=1}^{N} \sqrt{{\widehat{v_{x_{i}}}}^{2}+{\widehat{v_{y_{i}}}}^{2}}
$$

4.1.5. Mean Standard Deviation of Estimated Modulus. We introduced the mean standard deviation of the modulus estimates in (20):

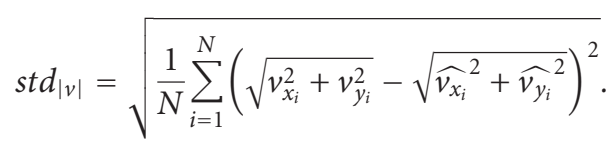

4.1.6. Mean Orientation of the Velocity Vector. The last characteristic is the mean estimated orientation of the velocity vector, defined in (21):

$$
\hat{\varphi}=\frac{1}{N} \sum_{i=1}^{N} \arctan \left(\frac{\widehat{v_{y_{i}}}}{\widehat{v_{x_{i}}}}\right) .
$$

4.1.7. Mean Standard Deviation of Orientation. Finally, the associated standard deviation is defined in (22):

$$
s t d_{\varphi}=\sqrt{\frac{1}{N} \sum_{i=1}^{N}\left[\arctan \left(\frac{v_{y_{i}}}{v_{x_{i}}}\right)-\arctan \left(\frac{\widehat{v_{y_{i}}}}{\widehat{v_{x_{i}}}}\right)\right]^{2}} .
$$

4.2. Results. The filters used for this evaluation were defined over a neighbourhood of $21^{*} 21^{*} 21$ voxels and an angular resolution equal to $2^{\circ}$ for $\theta$ and $5^{\circ}$ for $\varphi$. The energy criteria were calculated over a window of $7^{*} 7^{*} 7$ voxels by setting $C=6, R=6$, and $F=6$.

We constructed several curves from the performance criteria that were computed for each sequence. Figures 9 and 10 represent the normalized mean error and the normalized mean standard deviation along $x$ and $y$, respectively. Figure 11 illustrates the mean velocities estimated inside the vessel, $\widehat{v_{x}}$ and $\widehat{v_{y}}$, compared with theoretical mean velocities, represented with black lines. Finally, Figure 12 shows the mean modulus and orientations with their associated standard deviations.

According to Figure 9, the mean normalized error along $x$ does not rise above $15 \%$. We note that the best estimates are obtained for an angle $\varphi=0^{\circ}$. By observing how this error evolves, we see that it globally rises in the same way as the orientation. Moreover, the estimates from simulated sequences appear to be slightly better than the estimates from the experimental sequences. Indeed, it should be 


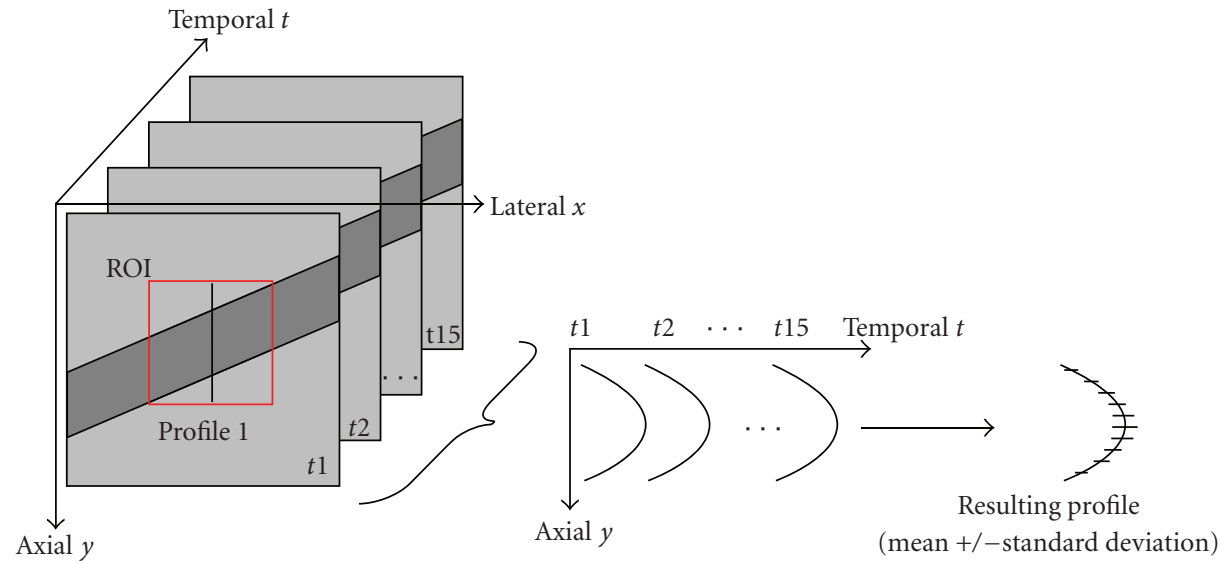

Figure 8: Computation of profiles (mean \pm standard deviation) from estimates on 15 successive frames.

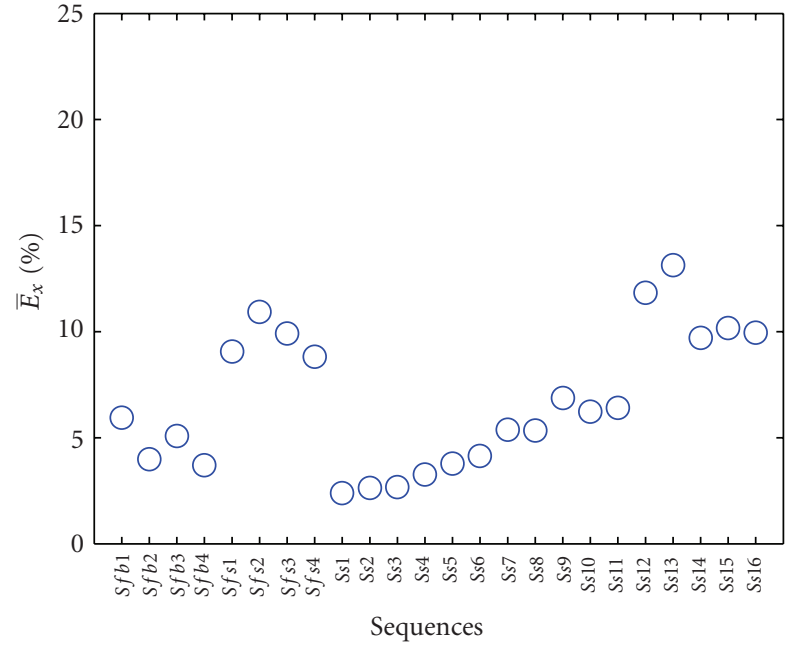

(a)

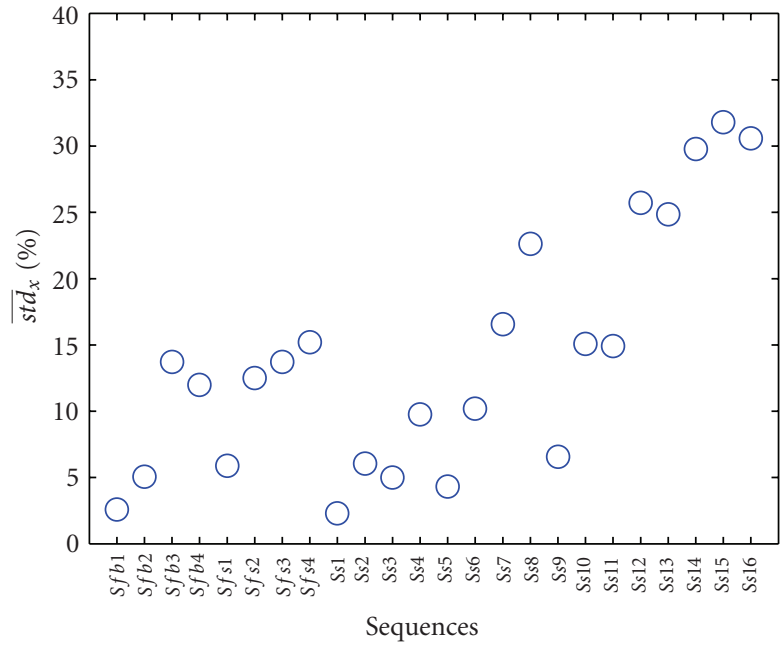

(b)

FIGURE 9: For each sequence, (a) normalized mean error $\overline{E_{x}}$, (b) normalized mean standard deviation $\overline{s t d_{x}}$.

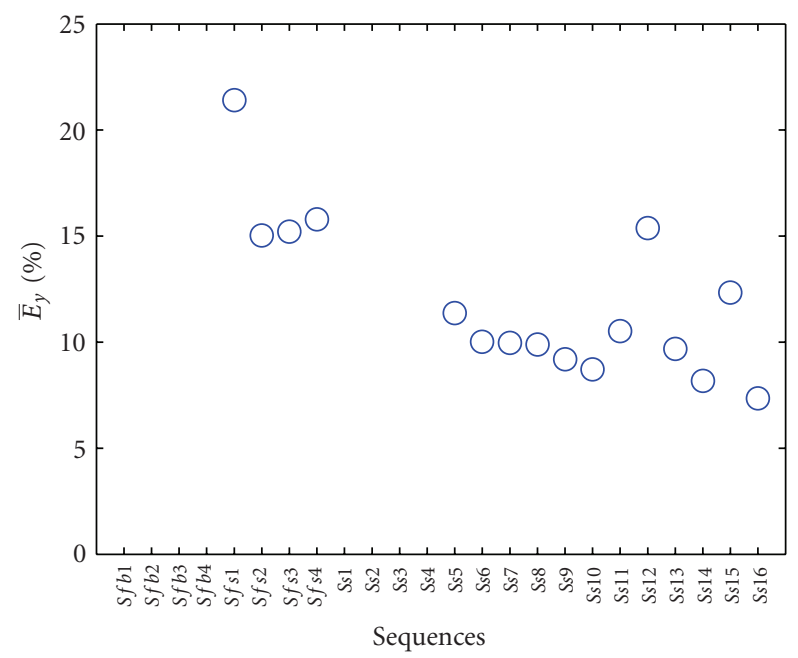

(a)

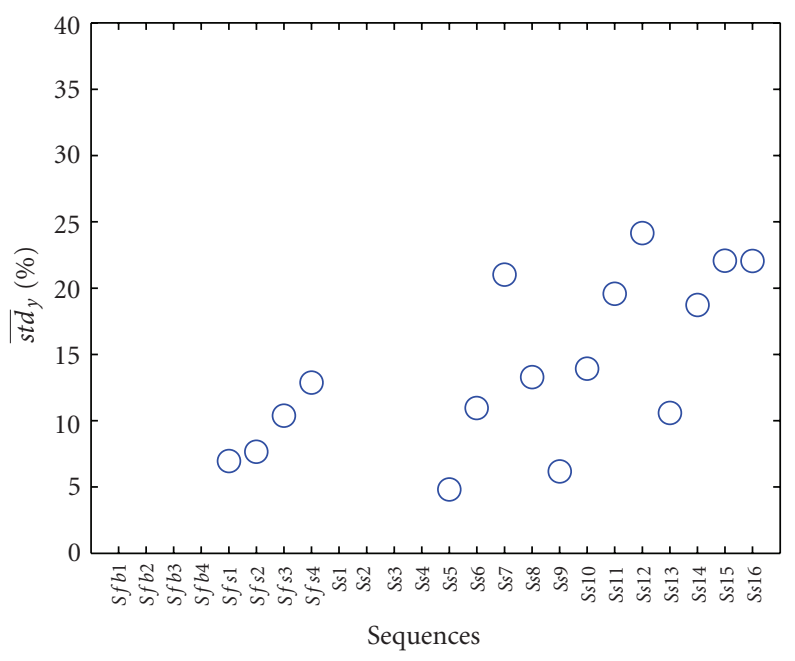

(b)

FIGURE 10: For each sequence, (a) normalized mean error $\overline{E_{y}}$, (b) normalized mean standard deviation $\overline{s t d_{y}}$. 


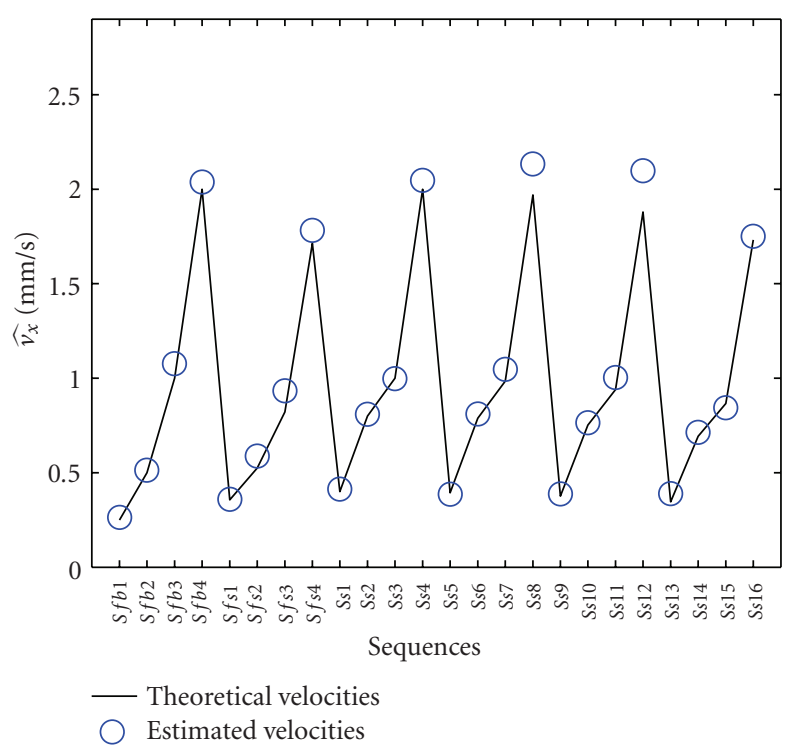

(a)

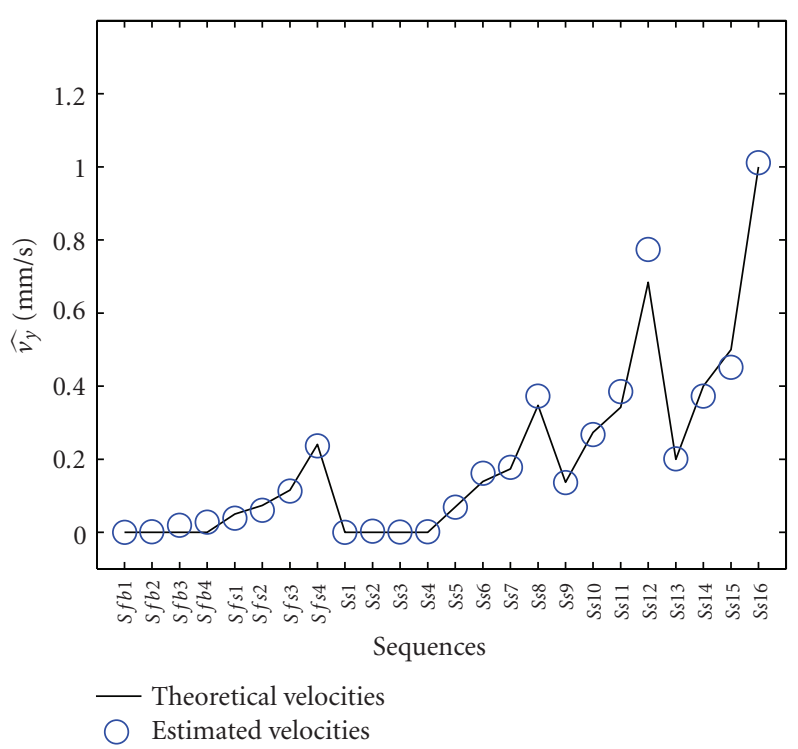

(b)

FIGURE 11: For each sequence, (a) estimated mean velocity $\widehat{v_{x}}$, (b) estimated mean velocity $\widehat{v_{y}}$.

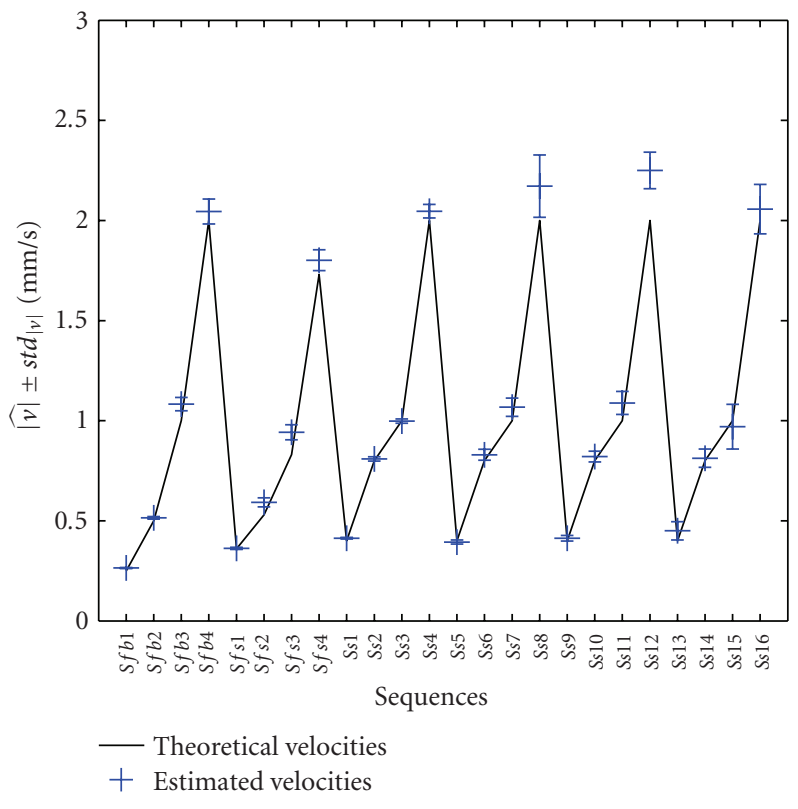

(a)

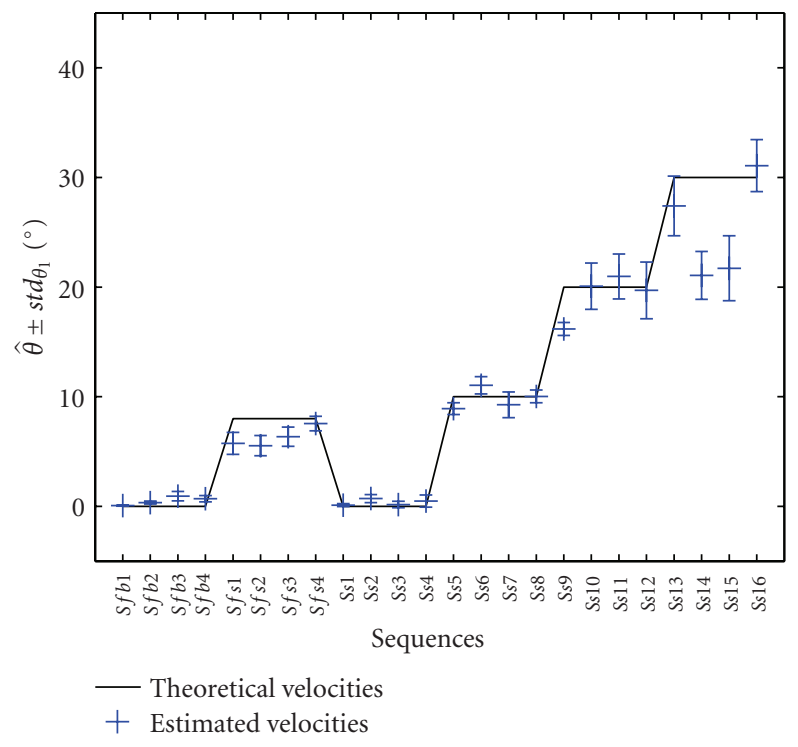

(b)

FIGURE 12: For each sequence, (a) mean and standard deviations of the mean velocity vector modulus $\mid \widehat{v \mid} \pm s t d_{|v|}$ (b) mean and standard deviations of mean velocity vector orientation $\hat{\varphi} \pm s t d_{\varphi}$.

noted that estimates from experimental sequences $S_{f s 1-4}$ are less accurate. Let us add that the theoretical values for experimental sequences are not an absolute reference. Concerning standard deviations along $x$, it is difficult to draw a conclusion. However, we can assert that an increase in the mean velocity generates an increase in the normalized standard deviations that reach up to $30 \%$ in the case of the maximum orientation equal to $30^{\circ}$. Once again, we can note that the lowest standard deviations were obtained with longitudinal flows, situations where Doppler methods fail to provide estimates.

The curves presented in Figure 10 are slightly different from the others because they do not contain values corresponding to sequences $S_{f b 1-4}$ and $S_{s 1-4}$. Indeed, normalization is not possible for these cases because $v_{\max }=0$. Estimates from sequences $S_{f s 1-4}$ exhibit the highest values for $\overline{E_{y}}$, between $15 \%$ and $20 \%$. Otherwise, the mean errors along $y$ are located around $10 \%$, with two extrema values for 
$S_{s 12}$ and $S_{s 15}$. The trend seems to be inverted in comparison with the previous curve of $\overline{E_{x}}$. The standard deviation values $\overline{s t d_{y}}$ are slightly less than those with $\overline{s t d_{x}}$. Moreover, the normalized standard deviations increase when velocity increases.

The curves presented in Figure 11 are relevant to a global analysis of the estimator's quality. Contrary to criteria $\overline{E_{x}}$ and $\overline{E_{y}}$, which provide local information, the mean values estimated evaluate whether or not an estimation is acceptable. Blood velocity estimation methods rarely provide dense velocity fields. Concerning $\widehat{v_{x}}$, it can be noted that the less accurate estimates are those from sequences with a high mean velocity. This underlines the saturation phenomenon, due to the use of the tangent function to calculate velocity modulus.

Errors are more often due to overestimations than underestimations. However, future systems with a higher frame rate would correct this small limitation. Concerning $\widehat{v_{y}}$, the errors compared to the theoretical velocity values are again limited. However, contrary to $\widehat{v_{x}}$, an underestimation phenomenon appears for some sequences. We also note that null components $v_{y}=0$ of sequences $S_{f b 1-4}$ and $S_{s 1-4}$ are always nearly perfectly estimated. Finally, we observe that the mean velocities are globally close to the theoretical components.

The last two curves in Figure 12 are also pertinent to obtaining global information on the method's behaviour. The first (Figure 12(a)) is a representation of the modulus mean value plus or minus the standard deviation. Logically, it shows an increase in the standard deviation when the modulus increases because there is no normalization. This curve also confirms the comments previously made on the mean components $\hat{v}_{x}$ and $\hat{v_{x}}$. The increase in errors is correlated to the increase in mean velocities and the errors are mainly due to overestimations. Regarding the mean orientations, Figure 12(b) contains the estimated mean values plus or minus the standard deviations. It is clear that orientations are not estimated as well as the modulus. The errors obtained for velocity components along $x$ and $y$ seem to be related to the errors on $\varphi$ rather than the errors on $\widehat{|v|}$. Thus, the orientation is systematically underestimated for sequences $S_{f s 1-4}$. Moreover, important errors are also denoted for sequences $S_{f_{s 14}}$ and $S_{f s 15}$. In addition, the orientations estimated for sequences with $10^{\circ}$ and $20^{\circ}$ are correct. Finally, let us add that the estimates of null orientations, for longitudinal flows, are globally the best.

To conclude on this part, we assert that the results obtained with our method are good compared to theoretical values and to estimates obtained with other blood velocity estimation methods [21]. They provide good information on the global behaviour of the flows. We have notably seen that the estimated mean velocities, the modulus, and the projected components were all close to theoretical values with low variability. Locally, estimates can appear less precise, with errors exceeding 10\% along $x$ and $20 \%$ along $y$. Similarly, local variability is increased with the normalized standard deviation reaching $20 \%$. We also observe an increase of the normalized standard deviations when the mean velocities are the highest. These comments underline the different degrees of quality of the estimates, viewed from a local or global point of view. Let us add that blood velocity estimation methods such as Doppler methods do not provide dense velocity fields. It should be remembered that the sequences contained flows whose orientations were less than $30^{\circ}$, with regards to lateral direction, because these cases are not solved with classical Doppler techniques.

\section{Conclusion}

In this paper, we proposed an original approach to estimate vector velocity from ultrasound image sequences. Our approach is based on a bank of 3D spatiotemporal quaternionic filters to estimate local texture orientations. It evaluates displacements in pixels per frame by estimating texture orientations within a $3 \mathrm{D}(2 \mathrm{D}+\mathrm{t})$ spatiotemporal volume. The method only requires textured data and is not dedicated to high-frequency imaging. However, this kind of data has better resolution and so texture is finer. The method was evaluated with a large set of experimental and simulated flow sequences. We studied sequences with different mean velocities whose velocity distributions followed parabolic laws. This kind of data is of great interest since it can evaluate displacements from zero pixels at the border of the vessel up to several pixels at the center. However, this type of data remains difficult to study because each pixel at each depth has a different velocity.

The results underlined several positive aspects of the method presented, notably the method's good behaviour in characterizing the flows studied. However, the results also demonstrated that obtaining accurate local estimates is very challenging; this difficulty is shared by all the approaches dealing with this problem. A possible explanation, with our proposal, can be related to the axial filter kernel size. Indeed, filters cover several voxels along depth, whereas the parabolic profile generates different velocities as a function of the depth within the vessel. This phenomenon introduces ambiguity on velocity.

Moreover, the range of displacements that can be estimated with our proposal is directly related to the intrinsic definition of the spatiotemporal approach, and particularly to the use of the tangent function. The range of potential velocities then depends on the frame rate and on the spatial sampling. Thus, estimation of displacements greater than six pixels per frame leads to greater errors, assuming fixed spatial and temporal sampling. Errors can be reduced by increasing the frame rate or decreasing the spatial sampling. In a general way, we showed that spatiotemporal quaternionic filters are a good alternative to Doppler methods for dense motion field estimation in ultrasound imaging. Note that this paper did not investigate in vivo data, which is one of the main perspectives to this work.

\section{Acknowledgments}

The authors wish to thank S. Foster and A. Needles from Sunnybrook Health Sciences Center, Toronto, Canada, where 
the experiments were conducted. They also thank the French Ministry of Higher Education and Research for financial support.

\section{References}

[1] F. S. Foster, M. Y. Zhang, Y. Q. Zhou et al., "A new ultrasound instrument for in vivo microimaging of mice," Ultrasound in Medicine and Biology, vol. 28, no. 9, pp. 1165-1172, 2002.

[2] C. Kasai, K. Namekawa, A. Koyano, and R. Omoto, "Real-time two-dimensional blood flow imaging using an autocorrelation technique," IEEE Transactions on Sonics and Ultrasonics, vol. 32, no. 3, pp. 458-464, 1985.

[3] O. Bonnefous and P. Pesqué, "Time domain formulation of pulse-Doppler ultrasound and blood velocity estimation by cross correlation," Ultrasonic Imaging, vol. 8, no. 2, pp. 73-85, 1986.

[4] S. G. Foster, P. M. Embree, and W. D. O’Brien Jr., "Flow velocity profile via time-domain correlation: error analysis and computer simulation," IEEE Transactions on Ultrasonics, Ferroelectrics, and Frequency Control, vol. 37, no. 3, pp. 164175, 1990.

[5] P. M. Embree and W. D. O’Brien, "Volumetric blood flow via time-domain correlation: experimental verification," IEEE Transactions on Ultrasonics, Ferroelectrics, and Frequency Control, vol. 37, no. 3, pp. 176-189, 1990.

[6] K. W. Ferrara and V. R. Algazi, "A new wideband spread target maximum likelihood estimator for blood velocity estimation-I: theory," IEEE Transactions on Ultrasonics, Ferroelectrics, and Frequency Control, vol. 38, no. 1, pp. 1-16, 1991.

[7] J. A. Jensen and P. Munk, "A new method for estimation of velocity vectors," IEEE Transactions on Ultrasonics, Ferroelectrics, and Frequency Control, vol. 45, no. 3, pp. 837-851, 1998.

[8] E. I. Céspedes, C. L. De Körte, and A. F. W. van der Steen, "Echo decorrelation from displacement gradients in elasticity and velocity estimation," IEEE Transactions on Ultrasonics, Ferroelectrics, and Frequency Control, vol. 46, no. 4, pp. 791801, 1999.

[9] W. Aoudi, H. Liebgott, A. Needles, V. Yang, F. S. Foster, and D. Vray, "Estimation methods for flow imaging with high frequency ultrasound," Ultrasonics, vol. 44, pp. e135-e140, 2006.

[10] V. L. Newhouse, K. S. Dickerson, D. Cathignol, and J.-Y. Chapelon, "Three-dimensional vector flow estimation using two transducers and spectral width," IEEE Transactions on Ultrasonics, Ferroelectrics, and Frequency Control, vol. 41, no. 1, pp. 90-95, 1994.

[11] P. Tortoli, G. Bambi, and S. Ricci, "A novel dual-beam approach for removing Doppler angle ambiguity," in Proceedings of the IEEE Ultrasonics Symposium, pp. 150-153, Rotterdam, The Netherlands, 2005.

[12] L. N. Bohs, B. J. Geiman, M. E. Anderson, S. C. Gebhart, and G. E. Trahey, "Speckle tracking for multi-dimensional flow estimation," Ultrasonics, vol. 38, no. 1, pp. 369-375, 2000.

[13] A. Basarab, H. Liebgott, F. Morestin et al., "A method for vector displacement estimation with ultrasound imaging and its application for thyroid nodular disease," Medical Image Analysis, vol. 12, no. 3, pp. 259-274, 2008.

[14] L. S. Wilson and R. W. Gill, "Measurement of two-dimensional blood velocity vectors by the ultrasonic speckle projection technique," Ultrasonic Imaging, vol. 15, no. 4, pp. 286-303, 1993.
[15] N. Oddershede, L. Løvstakken, H. Torp, and J. A. Jensen, "Estimating 2-D vector velocities using multidimensional spectrum analysis," IEEE Transactions on Ultrasonics, Ferroelectrics, and Frequency Control, vol. 55, no. 8, pp. 1744-1754, 2008.

[16] A. Marion and D. Vray, "Spatiotemporal filtering of sequences of ultrasound images to estimate a dense field of velocities," Pattern Recognition, vol. 42, no. 11, pp. 2989-2997, 2009.

[17] J. Meunier and M. Bertrand, "Echographic image mean gray level changes with tissue dynamics: a system- based model study," IEEE Transactions on Biomedical Engineering, vol. 42, no. 4, pp. 403-410, 1995.

[18] A. Marion and D. Vray, "Toward a real-time simulation of ultrasound image sequences based on a $3 \mathrm{D}$ set of moving scatterers," IEEE Transactions on Ultrasonics, Ferroelectrics and Frequency Control, vol. 56, no. 10, pp. 2157-2179, 2009.

[19] K. V. Ramnarine, D. K. Nassiri, P. R. Hoskins, and J. Lubbers, "Validation of a new blood-mimicking fluid for use in Doppler flow test objects," Ultrasound in Medicine and Biology, vol. 24, no. 3, pp. 451-459, 1998.

[20] F. Gran and J. A. Jensen, "Directional velocity estimation using a spatio-temporal encoding technique based on frequency division for synthetic transmit aperture ultrasound," IEEE Transactions on Ultrasonics, Ferroelectrics, and Frequency Control, vol. 53, no. 7, pp. 1289-1299, 2006.

[21] A. Marion, W. Aoudi, A. Basarab, P. Delachartre, and D. Vray, "Blood flow evaluation in high-frequency, $40 \mathrm{MHz}$ imaging: a comparative study of four vector velocity estimation methods," Elsevier Ultrasonics, vol. 50, no. 7, pp. 683-690, 2010. 